\title{
Gross pathology and histopathology in fish: summary
}

\author{
A. D. Vethaak \\ Ministry of Transport and Public Works, Public Works Department, Tidal Waters Division, Ecotoxicology Section,
} PO Box 20906, 2500 EX The Hague, The Netherlands

\section{INTRODUCTION}

The principal aim of the group of papers on gross pathology and histopathology was to assess the value of epidemiological studies of disease in dab Limanda limanda and of quantitative histological indices in the same species, for monitoring environmental quality in offshore waters.

The Bremerhaven Workshop provided a unique opportunity for a comparative study, with a comprehensive chemical programme as a background, and with a variety of other techniques being used to study biochemical and molecular/cellular effects in the same dab population.

The occurrence of the following parameters was quantified at 6 stations representing a known pollution gradient in the German Bight: (1) four externally visible diseases and gross liver nodules (with associated confirmatory histological diagnosis); (2) histopathologically identified lesions in livers without gross nodules; (3) melanomacrophage centres (MMCs) in the spleen.

The first two of these categories are dealt with in a single paper, which in view of the extensive amount of fieldwork involved was of necessity a team effort involving all participants. The third category is closely related to the first two, but has a paper to itself.

The studies of the group were focused on a single species (dab) in a specific area (the German Bight), with sampling during one particular month of the year (March). The results cannot necessarily be applied more generally to other species, areas or seasons in the North Sea.

It may be useful here to outline some of the limitations of the epidemiological approach to fish disease research. It is normally dependent on labour-intensive use of research vessels, often in poor working conditions, and does not allow the use of complex laboratory techniques. Furthermore, if epidemiological data are to be accurately interpreted, they must be based on extensive and continued sampling lasting several years, and on knowledge about the biology of the populations studied, particularly their movement patterns over long periods of time. To a certain extent, therefore, the interpretation of the data collected during this study has to depend largely on relevant published findings.

\section{EPIDEMIOLOGY OF EXTERNALLY VISIBLE DISEASES AND GROSS LIVER NODULES}

Two replicate surveys were aimed at defining spatial variation in disease prevalence along the pollution gradient, and at establishing relationships with contaminant concentrations and selected other potential causal factors. Of the 4 external diseases (lymphocystis, epidermal hyperplasia/papilloma, skin ulcerations (acute and healing stages), and X-cell gill lesion), the findings of the surveys, together with published evidence, suggest that epidermal hyperplasia/papilloma seems to be the most promising for biological effects monitoring. The prevalence of this disease shows general agreement with the chemical data, showing decreasing levels in an offshore direction from Stn 3 to 9 . The other 3 diseases showed no clear trend, or in the case of lymphocystis, the opposite trend.

Gross liver nodules $\geq 2 \mathrm{~mm}$ in diameter were found at most of the stations, and histological investigation revealed that $57.5 \%$ of them represented neoplastic and putative pre-neoplastic lesions. Smaller liver nodules and other lesions detected during gross examination did not represent neoplastic lesions, but 
about half the cases corresponded to putative preneoplastic lesions. No spatial trends could be established for these histologically identified lesions due to their low prevalences. This was probably related to a scarcity of large fish, which have the highest prevalences of liver nodules. The data do confirm, however, the importance of histological confirmation of lesions identified during gross examination.

The epidemiological study also provided a test of the published ICES guidelines for the implementation of fish disease surveys. It appears from the present data that some of the recommended minimum sample sizes are too small. On the other hand, the observed scarcity of large fish at certain stations makes it impractical to attain the minimal sample sizes, thus confounding any attempts to establish spatial trends for liver neoplasia.

\section{HISTOPATHOLOGICAL INVESTIGATION OF APPARENTLY HEALTHY LIVERS}

Some of these livers were found to bear putative preneoplastic lesions, but again low prevalences together with small sample sizes prevented the establishment of any spatial trends in relation to pollution.

\section{MELANOMACROPHAGE CENTRES (MMCS) IN THE SPLEEN}

The approach consisted of investigating spatial variability in the occurrence of haemosiderin, a cellular breakdown product found in MMCs, in sections of dab spleens. Attempts were then made to relate this variability to the presence of external diseases and to contaminant concentrations in the liver.

Haemosiderin occurrence was lowest at Stns $3 \& 9$ at either end of the transect. Because MMCs may be a possible indicator of the function of the non-specific immune system, these findings could be interpreted as suggesting a contamination-associated immunosuppressive effect at these 2 stations. On the other hand, the spatial pattern does not correspond to the observed pollution gradient and at the same time, the index based on MMCs did not appear to be linked to the general health of the fish, as measured by disease prevalences.

Due to the limited scope of the present study, sup- porting tests will be necessary to investigate the immunological significance of MMCs in spleen. The slow accumulation of haemosiderin within MMCs, coupled with effects of fish migration, may make this technique inappropriate for measuring short-term stress at specific sampling sites.

\section{CONCLUDING REMARKS}

Despite the apparent promise of epidermal hyperplasia/papilloma for biological effects monitoring, its aetiology, though probably infectious, remains uncertain and further studies and experiments are needed to clarify its significance as a pollution indicator.

The inconclusive findings regarding liver neoplasia and associated lesions do not justify their exclusion from future monitoring studies. There is substantial evidence in the literature that similar lesions in other flatfish species, especially those inhabiting North American waters, have a chemical-related cause. However, further evidence is needed to establish a causal relationship in dab. It could be argued that the occurrence of liver neoplasia in North Sea dab indicates exposure to carcinogenic compounds, thereby representing one of the few critical toxicological end points proposed to date for the marine environment. Results obtained by other techniques deployed at the workshop show clear biochemical effects and early indications of changes in liver function which correspond well with the known pollution gradient; these changes could ultimately lead to liver diseases such as neoplasia. It remains possible, however, that the chronic nature of liver neoplasia, coupled with the migratory behaviour of the target species and the possible elimination of diseased individuals from the population, makes the disease inappropriate for measuring toxic effects. Nevertheless, liver neoplasia would still have toxicological and ecological significance.

Information in several areas, essential to establishing the validity of an epidemiological approach, is needed before any of the above conditions can be adopted as reliable indicators. These areas include the migratory patterns of North Sea dab populations, the natural background levels of disease in the absence of pollution, and the existence of cause-and-effect relationships which can only be elucidated by experimental work 